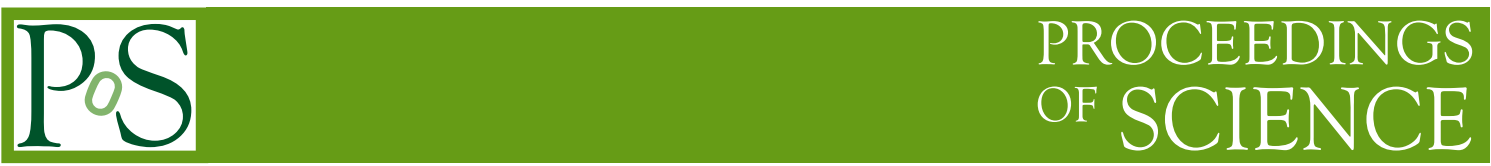

\title{
LHCb Upgrade - The Scintillating Fibre Tracker
}

\section{B. D. Leverington*}

Ruprecht-Karls-Universitaet, Heidelberg, Germany

E-mail: leverington@physi.uni-heidelberg.de

on behalf of the LHCb Collaboration

The LHCb detector will be upgraded during 2019-2020 in order to collect data from proton-proton collisions at the LHC at higher instantaneous luminosities and to read out the data at $40 \mathrm{MHz}$ using a trigger-less read-out system. All front-end electronics will be replaced and several subdetectors must be redesigned to cope with the higher occupancy. The current tracking detectors downstream of the LHCb dipole magnet will be replaced by the Scintillating Fibre (SciFi) Tracker. The SciFi Tracker will be constructed using $2.5 \mathrm{~m}$ long scintillating fibres and read out by Silicon Photomultipliers (SiPM) located outside the acceptance. The fibres have a diameter of $0.25 \mathrm{~mm}$, are wound into ribbons with 5 or 6 staggered layers of fibres, and will cover a total active area of around $360 \mathrm{~m}^{2}$. State-of-the-art multi-channel SiPM arrays are being developed to read out the fibres. A custom ASIC, the PACIFIC, will be used to digitise the signals from the SiPMs and additional front-end electronics based on FPGAs will be used to reconstruct hit positions. There are a number of challenges involved in the construction of this detector: the radiation hardness of the fibres and the SiPMs; the mechanical precision required while building large active detector components; and the cooling required to mitigate the effects of radiation damage. The evolution of the design since the Technical Design Report in 2014 will be presented.

The European Physical Society Conference on High Energy Physics

22-29 July 2015

Vienna, Austria

${ }^{*}$ Speaker. 


\section{Introduction}

The $\mathrm{LHCb}$ detector is a single-arm forward spectrometer designed for studying particles containing $b$ or $c$ quarks. A full description of the LHCb detector can be found in Ref. [1]. As of yet, in LHCb, and at the LHC in general, no New Physics has been discovered, though some smaller deviations from theory have been seen. LHCb is only limited from improving on these measurements by statistics and not systematic uncertainties. With this in mind, an upgrade to the LHCb spectrometer has been proposed in order to collect 100 times more data than has been analysed so far in order to reduce the uncertainties of the measurements by a factor of ten such that they are equivalent to theoretical uncertainties [2,3].

In 2012, the trigger rate has been brought to its maximum allowable value, such that a further increase in the rate of collected events is not possible. Data are currently recorded at an instantaneous luminosity of $4 \cdot 10^{32} \mathrm{~cm}^{-2} \mathrm{~s}^{-1}$, a factor of two above the design value, but moving beyond this does not increase signal yields in hadronic channels due to limitations in the hardware trigger. To increase the data collection rate beyond the $1 \mathrm{MHz}$ limit requires moving to a flexible software based trigger system[4] where events from each bunch crossing are selected in a computing farm and in turn requires all front-end electronics to be capable of $40 \mathrm{MHz}$ readout matching the proton collision rate. A total integrated luminosity of $50 \mathrm{fb}^{-1}$ will be collected during the upgrade running. Another limitation of the current detector preventing it from running at higher luminosities is the Outer Tracker. It has been shown that the Outer Tracker can run at up to 25\% occupancy $[2,3]$ before suffering a decrease in tracking efficiency. However, in the LHCb Upgrade scenario, where the luminosity is five times higher, the occupancy will reach $40 \%$ and above due to the coarse granularity of the $5 \mathrm{~mm}$ diameter straw drift tubes. The parts of the detector that perform insufficiently require a replacement by a detector with a finer granularity such that the high density of tracks in the detector near the beam pipe can be resolved.

Specific details regarding the upgrades to the other detectors can be found in the Letter of Intent [2], Framework TDR [3], and the specific detector Technical Design Reports available publicly. The most significant change to any of the detectors in the upgrade is replacing the current T-stations with a Scintillating Fibre (SciFi) based tracker, rather than modifying the existing detectors. This new tracker will be the focus of this Proceeding. A detailed description of the SciFi Tracker can be found in the Technical [5] and Engineering Design Reports [6].

\section{The Scintillating Fibre Tracker in the LHCb Upgrade}

The tracking detectors upstream and downstream of the LHCb dipole magnet are required to provide a high precision estimate of the momentum of charged particles. This leads to a precise mass resolution for decayed particles. There are three downstream tracking stations to provide standalone pattern recognition with a high efficiency together with high resolution in the bending plane of the magnetic field (horizontal). The tracking stations of the detector currently installed consist of two separate sub-detectors: the Outer Tracker (OT) and the Inner Tracker (IT). The OT was constructed using 5-mm diameter straw drift-tubes and covers around $99 \%$ of the $30 \mathrm{~m}^{2}$ detector surface. The IT is a silicon micro-strip detector with a strip pitch of $0.198 \mathrm{~mm}$ covering an area of $0.35 \mathrm{~m}^{2}$ in the high track density region around the beam-pipe. The SciFi Tracker is designed 

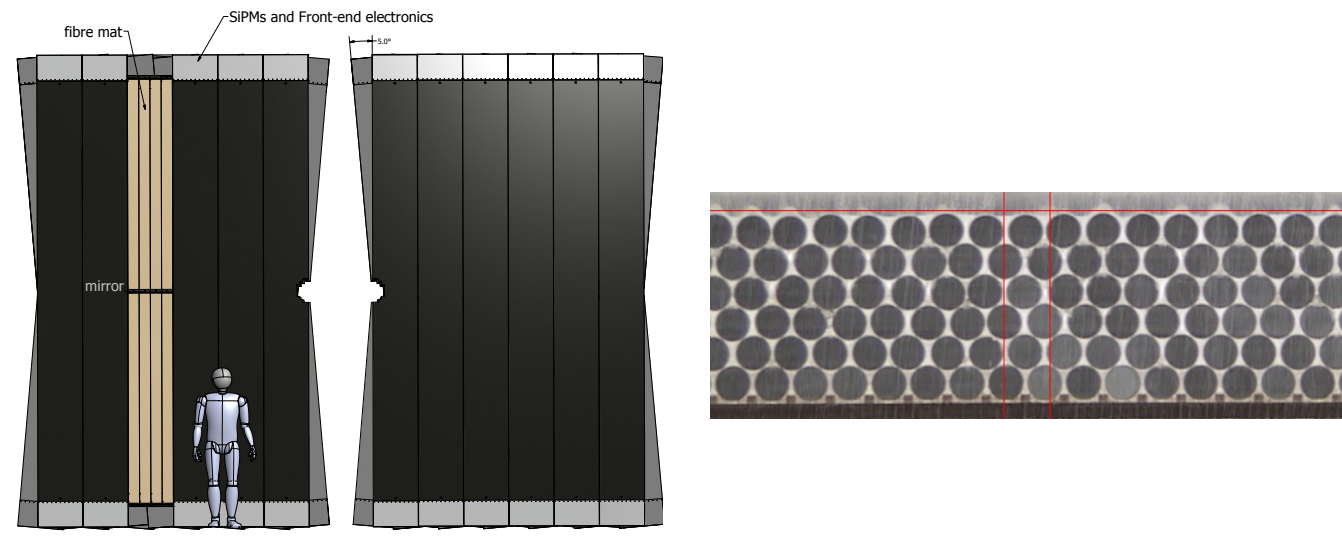

Figure 1: (left) 1 station of the SciFi tracker. Each station will have 4 layers $(X, U, V, X)$ in two halves oriented at $0,+5,-5$ and 0 degrees with respect to the vertical. Each layer will have 10-12 modules with eight mirrored fibre mats in each module. The SiPMs at the far end of the fibres outside of the acceptance. (right) A cross-section of a prototype fibre mat with six layers of fibres.

to replace these detectors and operate in the high rate environment with similar performance to the Inner Tracker while still covering the area of the Outer Tracker.

The new tracker will consist of three stations each with four detection planes arranged in an $\mathrm{X}, \mathrm{U}, \mathrm{V}, \mathrm{X}$ geometry. Each plane will have 10-12 modules. The fibres have a diameter of $0.25 \mathrm{~mm}$ and are wound into ribbons (mats) with six staggered layers. The modules will be constructed from $2.4 \mathrm{~m}$ long fibre mats and read out by multi-channel Silicon Photomultipliers (SiPMs) with a channel width of $0.25 \mathrm{~mm}$. The distance between the fibres in each layer is $0.275 \mathrm{~mm}$. The total active area that must be covered is around $360 \mathrm{~m}^{2}$ and over 10,000 $\mathrm{km}$ of fibres will be needed to construct the full detector with nearly 590k SiPM channels. This detector will face difficult challenges such as radiation damage to the sensitive components, as well as maintaining precision over such a large area during construction.

\subsection{Construction}

Amongst the many challenges for this detector is the actual construction of the sensitive detector modules for this large area, high precision detector. Continuous strands of scintillating fibre must be wound and bonded into a ribbon (or mat) with high precision and few defects. The cross section of a fibre mat can be seen in Fig. 1. The mats must then be straight as well as rigid in time between calibrations in the LHCb detector to better than $50 \mu \mathrm{m}$ in the horizontal and $300 \mu \mathrm{m}$ in the beam direction perpendicular to the module face. To achieve this, the winding process produces hardened epoxy pins that follow the central axis of a single fibre along the mats. The pins of eight mats are aligned in a $5 \mathrm{~m}$ long aluminium template and then bonded to a honeycomb/carbon-fibre composite panel. This construction method has been shown in the EDR [6] to produce large $5 \mathrm{~m}$ long, stable, and stiff detector modules with excellent precision.

\subsection{Scintillating Fibres}

The fibres are $0.250 \mathrm{~mm}$ in diameter, double clad with a polystyrene core, doped with two additional organic scintillators. Only one fibre type with the specifications needed for the experiment 
has been found so far, the SCSF-78MJ produced by Kuraray. The 78MJ fibre will typically produce several thousand photons per $\mathrm{MeV}$ deposited with a capture fraction of more than 5.35\% and has an average attenuation length greater than $3.25 \mathrm{~m}$ (the attenuation length is wavelength dependent). The wavelength spectra peaks at $435 \mathrm{~nm}$ near the source and extends into the green. This fibre type has a fast scintillation decay time of $2.8 \mathrm{~ns}$ or better. Nanostructured NOL based fibres are also under investigation and have been shown to have promising results, but are still being investigated [7].

It is expected from FLUKA simulations that the scintillating fibres will receive up to $35 \mathrm{kGy}$ of ionizing radiation in the regions closest to the beam-pipe after $50 \mathrm{fb}^{-1}$, as seen in Fig. 2. It has been seen that scintillating fibres will lose transmission [8] under irradiation. However, the literature describing this particular fibre is limited in the degree of damage in our radiation dose range. Multiple separate irradiation campaigns within the SciFi collaboration were undertaken to investigate the damage, irradiating the fibres with $24 \mathrm{GeV} / \mathrm{c}$ protons at the CERN-PS, $23 \mathrm{MeV}$ protons at the Karlsruhe Institute of Technology, in situ LHCb pit irradiation, as well as gamma and X-ray irradiation. The results are shown in Fig. 2 (left). The loss of transmission is rapid for even low doses but note the logarithmic horizontal axis. The large ionisation dose will be highly localised around the beam-pipe and therefore it is expected that the fibre tracker will be able to transmit enough of the scintillation light to the photodetectors to maintain a high hit efficiency, with a loss of only $40 \%$ of the signal in the worst region at the end of running. The scintillation production mechanism was not observed to be affected by irradiation. Studies of the radiation damage in the fibre are ongoing as this is one of the significant contributions to the loss in performance of the detector.
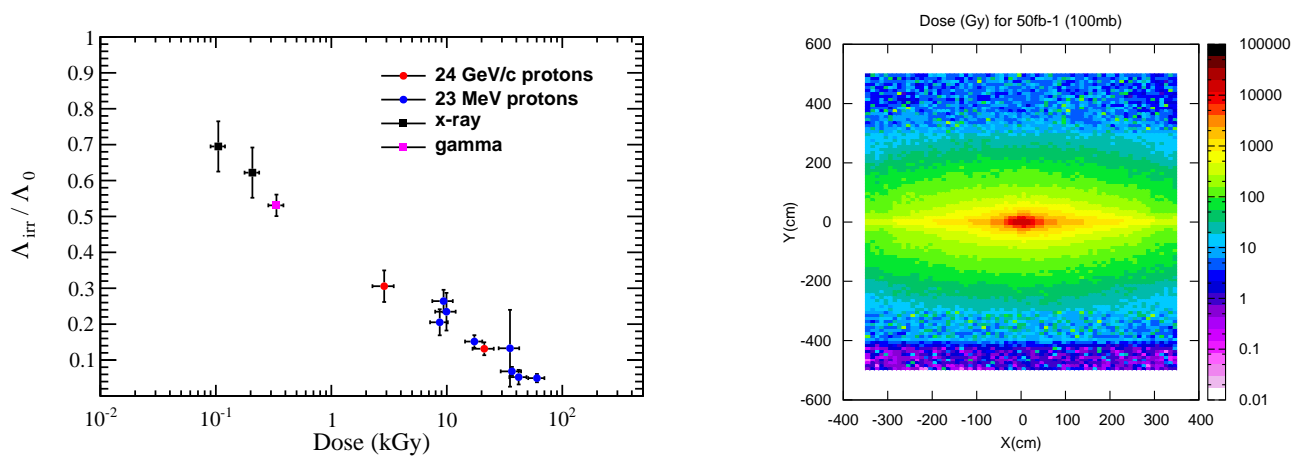

Figure 2: The ratio of attenuation length after irradiation to before as a function of dose for scintillating fibres for multiple source of ionizing radiation.

\subsection{Silicon Photomultipliers}

Silicon Photomultiplier technology has advanced considerably over the last couple of years with great improvement in reducing the inter-pixel crosstalk as well as increasing the photon detection efficiency (PDE). Previous devices had a typical crosstalk on the order of $21 \%$, but advances in trenching technology between the pixels has reduced the crosstalk to $10 \%$ levels currently on the latest Hamamatsu devices, with a larger geometrical fill factor increasing the maximum PDE 
to $39 \%$ at an operational overvoltage of $3.5 \mathrm{~V}$. The next generation of the arrays to be used for the tracker are expected to have crosstalk levels below 5\% with a PDE above 45\%. A total of 4096 128-channel arrays will be used in the upgrade, with an example device seen in Fig. 3.
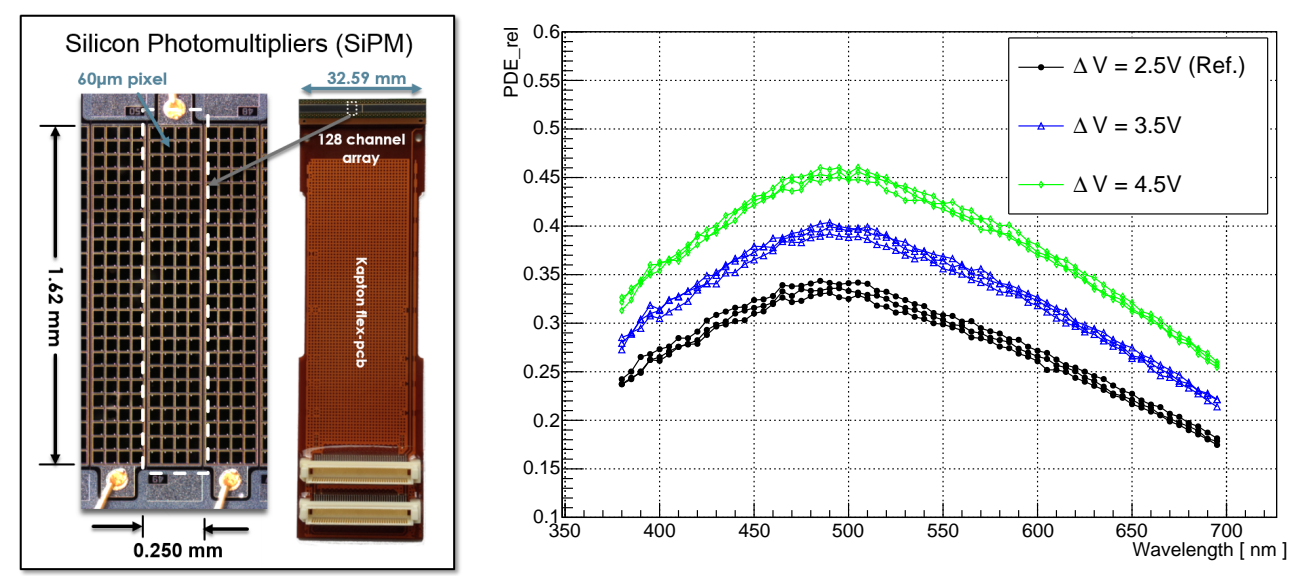

Figure 3: (left) A 128 channel SiPM array bonded to a Kapton flex-cable with a single channel close-up. (right) The PDE as a function of wavelength measured at different over-voltages using one of the latest Hamamatsu devices. Fig. taken from [9].

Like all silicon detectors, the SiPMs are subject to neutron and equivalent radiation damage which results in an increase of single pixel avalanches from thermal excitation. Pixel-crosstalk will in turn create larger amplitude signals which begin to resemble low amplitude signals. This is called the Dark Count Rate (DCR). The dark count rate (DCR) will increase with irradiation but can be reduced by lowering the operating temperature of the SiPMs. In the region of the SiPMs, a total neutron fluence of $6 \cdot 10^{11} 1 \mathrm{MeV} \mathrm{n}_{e q} \mathrm{~cm}^{-2}$ is expected after $50 \mathrm{fb}^{-1}$, which will require cooling the detectors to $-40^{\circ} \mathrm{C}$, resulting in a few $\mathrm{MHz}$ of dark signals. The DCR will reduce by a factor of two for every $10^{\circ} \mathrm{C}$, approximately. The small area of each SiPM channel, combined with fast read-out and shaping electronics, make it possible to operate the SiPMs at the single photo-electron level in the $\mathrm{LHCb}$ upgrade environment.

\subsection{Front-end Electronics}

The $40 \mathrm{MHz}$ trigger-less read-out in the LHCb upgrade will require zero-suppression of the data at the front-end electronics. A custom 64-channel front-end ASIC (PACIFIC) based on $130 \mathrm{~nm}$ CMOS TSMC technology is being developed. The PACIFIC consists of a pre-amplifier and a fast shaper, dual gated integrators to ensure zero dead-time, a track and hold stage, and a non-linear flash ADC consisting of three comparitors for applying signal thresholds for the digitisation, as seen in Fig. 4. Signals from the scintillating fibre mat will typically cover 2 or more channels with a mean amplitude of 12 or more photoelectrons after irradiation. Dark noise signals in the SiPM will typically be single photoelectrons with higher amplitudes coming from crosstalk, or random overlap in the integration stage of the electronics, but will be typically lower than 4 photoelectrons. The three thresholds in the flash ADC stage of the ASIC allow for identifying signal clusters and accurately determining their location while suppressing dark noise clusters. The digitised signals 
are then sent to an FPGA where individual channels are combined into clusters and the data is zero-suppressed before being sent to the computing farm.

\section{Conclusion}

The Scintillating Fibre tracker is crucial for the operation and success of the upgraded $\mathrm{LHCb}$ detector. The new detector will replace the current downstream tracking stations during LS2 in 2019/2020. The $0.250 \mathrm{~mm}$ diameter scintillating fibres, combined with the SiPMs and custom PACIFIC ASIC will provide fast signals for tracking and the new $40 \mathrm{MHz}$ software trigger. The ionising radiation damage to the scintillation fibres will reduce the signal amplitude, and nonionising damage to the SiPMs will create additional thermally induced noise signals. Sufficient scintillating fibre is installed to ensure a large enough signal to noise ratio, while the SiPMs will be cooled to manage the dark noise rate. Module production will begin at the beginning of 2016 with the final electronics being installed at the end of 2018. The detector will be ready for data taking in 2020

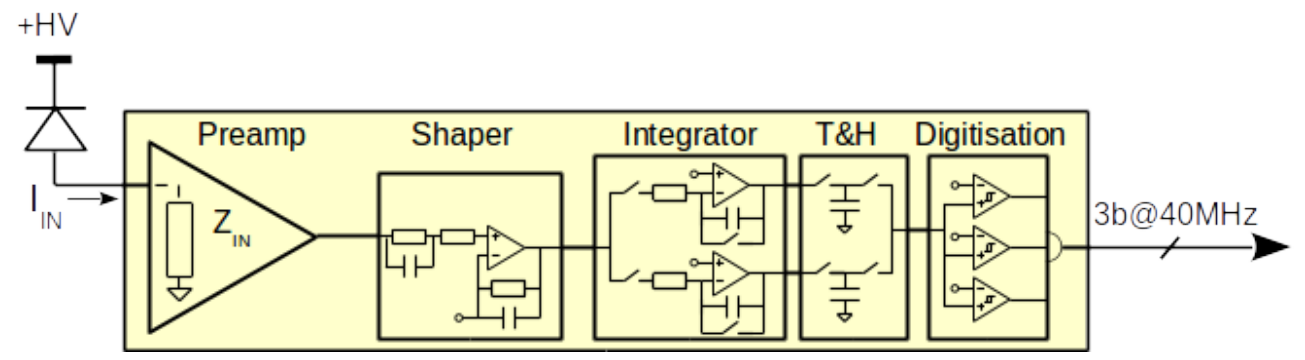

Figure 4: A single channel of the PACIFIC ASIC for the SciFi Tracker.

\section{References}

[1] LHCb collaboration, The LHCb Detector at the LHC, JINST 3 (2008) S08005.

[2] LHCb collaboration, Letter of Intent for the LHCb Upgrade, CERN-LHCC-2011-001, March 2011.

[3] LHCb collaboration, Framework TDR for the LHCb Upgrade, CERN-LHCC-2012-007, May 2012.

[4] LHCB collaboration, LHCb Trigger and Online Upgrade Technical Design Report, CERN-LHCC-2014-016, May 2014.

[5] LHCB collaboration, LHCb Tracker Upgrade Technical Design Report, CERN-LHCC-2014-001.

[6] LHCB collaboration, LHCb Scintillating Fibre Tracker Engineering Design Report: Fibres, Mats and Modules, CERN-LHCb-PUB-2015-008.

[7] Ponomarenko, Sergei A., et al. Nanostructured organosilicon luminophores and their application in highly efficient plastic scintillators, Scientific reports 4 (2014).

[8] C. Zorn, A Pedestrians Guide To Radiation Damage In Plastic Scintillators, Nuclear Physics B (Proc. Suppl.) 32 (1993) 377-383.

[9] Kuonen A., et al., Characterisation of the Hamamatsu MPPC multichannel array for LHCb SciFi Tracker, ,LHCb-INT-2015-14, April 2015 